\title{
Chitosan Oligosaccharide Based Hydrogel: an Insight into the Mechanical, Drug Delivery, and Antimicrobial Studies
}

\author{
Sarada Prasanna Mallick 1,*(D), Siva Prasad Panda 2(D), Abhinav Gayatri 1, Yashwanth Kunaal 1,

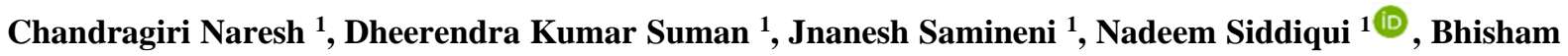 \\ Narayan Singh ${ }^{3}$
}

1 Department of Biotechnology, Koneru Lakshmaiah Education Foundation, Guntur, India-522502; yourssarada@kluniversity.in (S.P.M); abhinavgayatri@gmail.com (A.G.); ykunaalb1510@gmail.com (Y.K.); nareshsunny8975@gmail.com (C.N.); dheerendrasuman1@gmail.com (D.K.S.); saminenijnanesh@gmail.com (J.S.); siddiqui@kluniversity.in (N.S.);

2 College of Pharmacy, Koneru Lakshmaiah Education Foundation, Guntur, India-522502; sivaprasad.panda@kluniversity.in (S.P.P.);

3 School of Biochemical Engineering, Indian Institute of Technology (Banaras Hindu University), Varanasi, India-221005; bhisham6@gmail.com (B.N.S.);

* Correspondence: yourssarada@kluniversity.in;

Received: 18.08.2020; Revised: 5.10.2020; Accepted: 7.10.2020; Published: 14.10.2020

\begin{abstract}
Hydrogel is a macromolecular polymer gel constructed of a network of cross-linked polymer chains. Chitosan oligosaccharide (CO) is a natural polymer that has an efficient biodegradability and is used to prepare hydrogels for efficient drug delivery. $\mathrm{CO}$ is combined with Carboxy Methyl Cellulose (CMC) to produce a hydrogel in different compositions for the best suitable option where the drug delivery and biocompatibility are most efficient. The chemical hydrogels of the composition $\mathrm{CO}$ and $\mathrm{CMC}$ were prepared by adding the cross-linker mixture of glutaraldehyde, alcohol, and distilled water. After the hydrogels were prepared, it is subjected to a series of tests, i.e., microscopy, hemocompatibility, mechanical strength, impedance measurement, drug release, antimicrobial activity. Ccross-linking is achieved by adding a mixture of glutaraldehyde, alcohol, and distilled water. Thus, the best composition of the mixture of the two compounds - Chitosan oligosaccharide and the Carboxy Methyl Cellulose is determined and used for efficient drug delivery.
\end{abstract}

Keywords: hydrogel; chitosan oligosaccharide; carboxymethyl cellulose; hemocompatibility.

(c) 2020 by the authors. This article is an open-access article distributed under the terms and conditions of the Creative Commons Attribution (CC BY) license (https://creativecommons.org/licenses/by/4.0/).

\section{Introduction}

The materials of enthusiasm for this short survey are basically hydrogels, which are polymer organizes broadly swollen with water. Hydrophilic gels that are generally alluded to as hydrogels are systems of polymer chains that are some of the time found as colloidal gels in which water is the scattering medium $[1,2]$.

Analysts throughout the years, have characterized hydrogels in a wide range of ways. The most widely recognized of these is that hydrogel is a water-swollen and cross-connected polymeric system delivered by the straightforward response of at least one monomer. Another definition is that it is a polymeric material that displays the capacity to swell and hold a critical division of water inside its structure yet won't disintegrate in water. Hydrogels have gotten significant consideration in the previous fifty years, because of their remarkable guarantee an 
extensive variety of utilizations. They have added a level of adaptability fundamentally the same as regular tissue because of their extensive water content $[3,4]$.

The capacity of hydrogels to retain water emerges from hydrophilic utilitarian gatherings connected to the polymeric spine, while their protection from disintegration emerges from cross-interfaces between system chains. Numerous materials, both normally happening and engineered, fit the meaning of hydrogels [5]. In most recent two decades, common hydrogels were bit by bit supplanted by manufactured hydrogels, which has long administration life, the high limit of water assimilation, and high gel quality. Luckily, engineered polymers typically have very much characterized structures that can be adjusted to yield tailor capable degradability and usefulness. Hydrogels can be integrated from simply engineered segments. Additionally, it is steady in the states of sharp and solid variances of temperatures. As of late, hydrogels have been characterized as two-or multi-segment frameworks comprising of a threedimensional system of polymer chains and water that fills the space between macromolecules [6,7]. Contingent upon the properties of the polymer (polymers) utilized, and additionally on the nature and thickness of the system joints, such structures in a balance can contain different measures of water; normally, in the swollen express, the mass part of the water in a hydrogel is a lot higher than the mass portion of the polymer. Hydrogels might be combined in various "established" synthetic ways [8,9]. These incorporate one-advance methods like polymerization and parallel cross-connecting of multifunctional monomers, and additionally various advance strategies including the union of polymer particles having responsive gatherings and their resulting cross-connecting, perhaps at the same time by responding polymers with reasonable cross-connecting operators. The polymer designer can plan and integrate polymer systems with sub-atomic scale authority over a structure, for example, crossconnecting thickness and with custom-fitted properties, for example, biodegradation, mechanical quality, and compound and natural reaction to upgrades.

Chitosan oligosaccharide $(\mathrm{CO})$ is an oligomer of $\beta$ - $(1 \rightarrow 4)$ - connected d-glucosamine. $\mathrm{CO}$ can be set up from the deacetylation and hydrolysis of chitin, which is normally found in the exoskeletons of arthropods and bugs and the cell dividers of organisms [10,11]. Carboxymethyl cellulose (CMC) is a cellulose subordinate with carboxymethyl bunches ($\mathrm{CH}_{2}-\mathrm{COOH}$ )., usually utilized as its sodium salt, sodium carboxymethyl cellulose [12]. $\mathrm{CO}$ and CMC Salicylic Acid are a lipophilic monohydroxybenzoic acid, a sort of phenolic acid, and a $\beta$-hydroxy acid (BHA). The present article delineates the development and characterization of CO and CMC based chemical hydrogel using glutaraldehyde as a crosslinker. The delivery of the model antimicrobial agent (salicylic acid) and their antimicrobial efficiency has also been studied [13].

\section{Materials and Methods}

\subsection{Materials.}

Chitosan Oligosaccharide and Carboxy Methyl Cellulose were obtained from Sisco Research Laboratories Pvt. Ltd., Maharashtra, India. Ethanol was obtained from Honyon International Inc., Hong Yang Chemical Corp., China. Glutaraldehyde (GA) (25\%, for synthesis) was procured from Molychem, Mumbai, India, and Hydrochloric acid (35\% pure) was obtained from Merck Specialities Private Limited Mumbai, India. Salicylic acid (SA) was obtained from New Shakti Pharmaceuticals, Kanpur, U.P. Sodium citrate were procured from 
Loba Chemie, Mumbai, India. Goat blood was obtained from the local Butcher shop. Double distilled water was used throughout the study.

\subsection{Preparation of hydrogel.}

The preparations of hydrogels were carried out in two-step methodology. In the first step, the $\mathrm{CO}$ and $\mathrm{CMC}$ solutions being prepared. Physical hydrogels were prepared by varying the proportions of Chitosan Oligosaccharide and Carboxy Methyl Cellulose. The compositions of the hydrogels have been tabulated in Table 1 . The hydrogels were prepared by mixing CO and $\mathrm{CMC}$ and subsequently homogenizing the dispersion at $500 \mathrm{rpm}$ for $30 \mathrm{~min}$ at $40^{\circ} \mathrm{C}$. The homogenized mixture was then poured into culture bottles and stored under refrigerated conditions for further analysis. The chemical hydrogels were being prepared by adding crosslinker. Crosslinking agent (cross-linking is the formation of chemical links between molecular chains to form a three-dimensional network of connected molecules) consists of a mixture of Glutaraldehyde, $\mathrm{HCl}$, and Ethanol.

Chitosan oligosaccharide is combined with Carboxy Methyl Cellulose (CMC) to produce a hydrogel in different compositions for the best suitable option where the drug delivery and the biocompatibility are most efficient. Salicylic acid is used as the drug to carry out controlled drug delivery. The schematic representation of hydrogel preparation is shown in Figure 1.

\begin{tabular}{l|c|c|c|c}
\multicolumn{5}{c}{ Table 1. Composition of Hydrogels. } \\
Sample & $\begin{array}{c}\text { CO 2\% }(\mathbf{w} / \mathbf{v}) \\
(\mathbf{m l})\end{array}$ & $\begin{array}{c}\text { CMC 2\%(w/v) } \\
(\mathbf{m l})\end{array}$ & $\begin{array}{c}\text { Crosslinking } \\
\text { Agent }(\mathbf{m l})\end{array}$ & $\begin{array}{c}\text { Drug 1\% } \\
(\mathbf{w} / \mathbf{v})\end{array}$ \\
\hline S1 & - & 20 & 1.1 & \\
\hline S2 & 2 & 18 & 1.1 & - \\
\hline S3 & 4 & 16 & 1.1 & - \\
\hline S1D & - & 20 & 1.1 & $0.2 \mathrm{~g}$ \\
\hline S2D & 2 & 18 & 1.1 & $0.2 \mathrm{~g}$ \\
\hline S3D & 4 & 16 & 1.1 & $0.2 \mathrm{~g}$
\end{tabular}

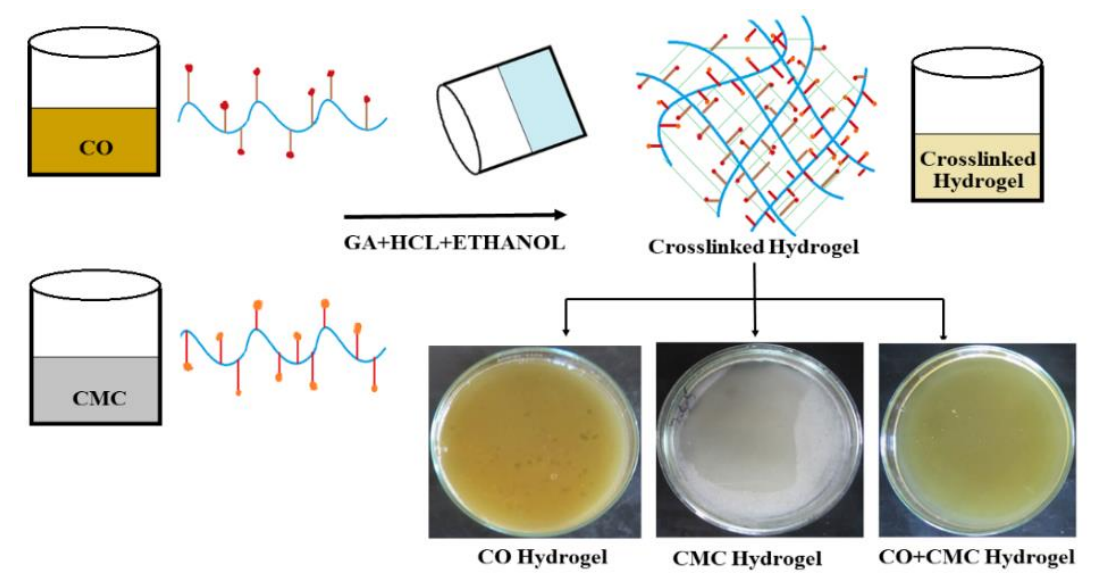

Figure 1. Schematic representation of hydrogel(s) preparation.

\subsection{Microscopy study.}

The microscopy studies of the prepared hydrogels were studied under a light microscope (LM-52-1803). Microscopy is the specialized field of utilizing magnifying instruments to see the microstructure of the hydrogels that cannot be seen with the unaided eye. 


\subsection{Hemocompatibility test.}

The hemocompatibility examinations were done as per the American Society for Testing and Materials standard procedure, which measured the amount of hemolysis in the sample's presence [14]. For this, fresh goat's blood was collected along with trisodium citrate (TSC) in the ratio of $3.8 \mathrm{~g}$ of TSC per $100 \mathrm{ml}$ to avoid coagulation. The citrated blood was diluted with normal saline in the ratio of $8: 10 ; 0.5 \mathrm{ml}$ of the diluted blood was taken in a centrifuge tube. Hydrogel samples (Chitosan Oligosaccharide + Carboxy Methyl Cellulose) of size $5 \mathrm{~mm} * 5 \mathrm{~mm}$ were put into the container, followed by $9.5 \mathrm{ml}$ of saline. For positive control, $0.5 \mathrm{ml}$ of diluted blood was mixed with $0.5 \mathrm{ml}$ of $0.01 \mathrm{~N} \mathrm{HCl}$ and subsequently diluted to $10 \mathrm{ml}$ with saline. $\mathrm{HCl}$ ruptured the red blood cells and released the hemoglobin into the solution. For negative control, $0.5 \mathrm{ml}$ of blood was diluted to $10 \mathrm{ml}$ with saline solution. The centrifuge tubes were incubated at $37^{\circ} \mathrm{C}$ for $60 \mathrm{~min}$. The optical density (OD) of all the solutions was measured at $545 \mathrm{~nm}$ using an ultraviolet (UV)-visible spectrophotometer. The formula for hemolysis (\%)

$$
\% \text { Hemolysis }=\text { ODtest }- \text { ODnegative } / \text { ODpositive }- \text { ODnegative } \times 100
$$

The percentage hemolysis was $<5$, then the material was considered as highly hemocompatible, a value $<10$ indicated hemocompatible, whereas a value $>20$ indicated nonhemocompatible.

\subsection{Mechanical analysis (compressive strength).}

The mechanical properties of the hydrogels were determined by compression using a static mechanical tester (CT3, Brookfield, USA). The hydrogels with different proportions of Chitosan Oligosaccharide and Carboxy Methyl Cellulose were used for the studies. A 30-mm flat probe was used for the studies.

\subsection{In vitro drug release study.}

Drug loaded hydrogel samples were used for the drug release. In vitro drug release study was carried out in the orbital shaker, briefly, drug loaded samples $(1 \mathrm{~cm} \times 1 \mathrm{~cm})$ were kept in $250 \mathrm{ml}$ of conical flasks containing $100 \mathrm{ml}$ phosphate buffer saline (pH 7.4) in an orbital shaker of $100 \pm 5 \mathrm{rpm}$ and was maintained to $37^{\circ} \mathrm{C}$. The experiment carried for a time interval of $6 \mathrm{~h}$. Five $\mathrm{ml}$ of samples were taken at a time interval of $30 \mathrm{~min}$ for $6 \mathrm{~h}$ and replaced with fresh PBS. After $6 \mathrm{~h}$ the replaced media were examined spectrophotometrically (UV Spectrophotometer, 22331, Hamburg, Eppendorf AG, USA) at $294 \mathrm{~nm}$ [15].

\subsection{Antimicrobial study.}

The antimicrobial efficiency of the hydrogels with different formulations against Gramnegative bacterium Escherichia coli (NCIM 5051) was performed [16,17]. The analysis was done by the bore-well method. Holes of $9 \mathrm{~mm}$ diameter were made into the agar plates using a SS steel borer. Hydrogels containing different proportions of Chitosan Oligosaccharide and Carboxy Methyl Cellulose are put into the bores with an equal amount in different locations of agar plates. The Petri-plates were incubated at $37 \pm 0.5^{\circ} \mathrm{C}$ for $24 \mathrm{~h}$ to allow the growth of the bacteria. The zone of inhibition was measured by using a ruler at the end of $24 \mathrm{~h}$. 


\section{Results and Discussion}

\subsection{Microscopy study.}

To investigate the microstructure of the hydrogels, we examined the morphology of the hydrogel using a light microscope. From Figure 2(a,c), we can observe the uniform morphology of the hydrogel due to only $\mathrm{CO}$ and $\mathrm{CMC}$; on the other hand, by mixing both the $\mathrm{CO}$ and $\mathrm{CMC}$ solutions (Figure 2(b)), the picture shows fiber and breaking like structure. The microscopy methodology was done in developing methods for understanding the drug release phenomenon from the hydrogel matrix [18].

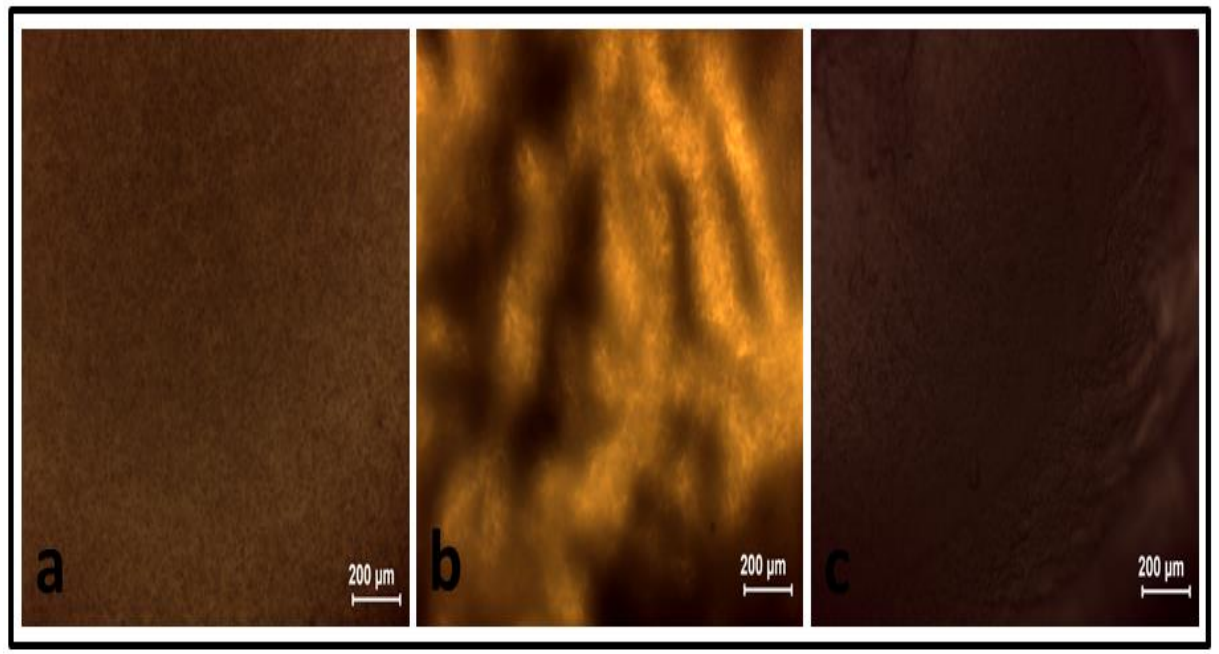

Figure 2. Light micrographs of Hydrogels: (a) S1, (b) S2, and (c) S3.

\subsection{Hemocompatibility test.}

Hemocompatibility of blood-contacting polymeric hydrogels is one of the most crucial criteria for their success in vivo methodology. Hemolytic activity of drug-loaded and standard hydrogels was performed as the measure of the extent of hemolysis that may be caused by the polymeric hydrogels against normal human erythrocytes. All samples loaded with drugs were found to be hemocompatible, out of which S1D was the most hemocompatible, followed by S2D and S3D. The results are shown in Table 2. If the percentage of hemolysis was $<5$, then the material was considered highly hemocompatible, and the value is <10 indicates hemocompatible; on the other hand, a value $>20$ indicates Non-hemocompatible [19].

Table 2. The percentage of hemolysis for the different compositions of hydrogels.

\begin{tabular}{l|c|c} 
Sl.No. & Sample ID. & \% Percentage hemolysis \\
\hline 1 & S1 & 2.3 \\
\hline 2 & S2 & 1.9 \\
\hline 3 & S3 & 3.5 \\
\hline 4 & S1D & 4.1 \\
\hline 5 & S2D & 3.9 \\
\hline 6 & S3D & 4.8
\end{tabular}

\subsection{Mechanical analysis.}

Current researches in polymeric hydrogels have been concentrating on mechanical strength improvement, ranging from the layout of microstructures to modification of compositions in hydrogels for proper drug release mechanisms. The compressive profiles of the hydrogels have been shown in Figure 3(a). It was observed that the compressive strength 
of the hydrogels was higher when the chitosan oligosaccharide proportion was more. This suggested that the chitosan oligosaccharide predominant hydrogels show higher mechanical strength.

\subsection{Drug release study.}

The high-water content of most hydrogels typically results in a relatively rapid release of drugs from the gel matrix over hours or days, particularly in the case of hydrophilic drugs for which hydrogel delivery is typically applied. The in vitro drug release profiles of salicylic acid from drug-loaded hydrogels have been shown in Figure 3(b). The rate of drug release depends upon the proportion of chitosan oligosaccharide and Carboxy Methyl Cellulose. A higher proportion of chitosan oligosaccharides shows a higher percentage of drug release. The drug release occurs very slowly in the composition S1D; this may be attributed to a higher water uptake rate.

\subsection{Antimicrobial study.}

The prepared Hydrogels were examined for its bactericidal action against Gramnegative E. coli through the agar diffusion process. Zone of inhibitions (ZOIs) found to be maximum for the S3D for the bacteria sample [20,21]. The ZOIs value for the bacterial samples follows the pattern S3D>S2D>S1D. Figure 3(c) shows the diameter of ZOIs for the gramnegative bacteria against the hydrogels [22]. These additionally recommend the bactericidal chapter of the prepared hydrogels guaranteeing its functions in drug release application.
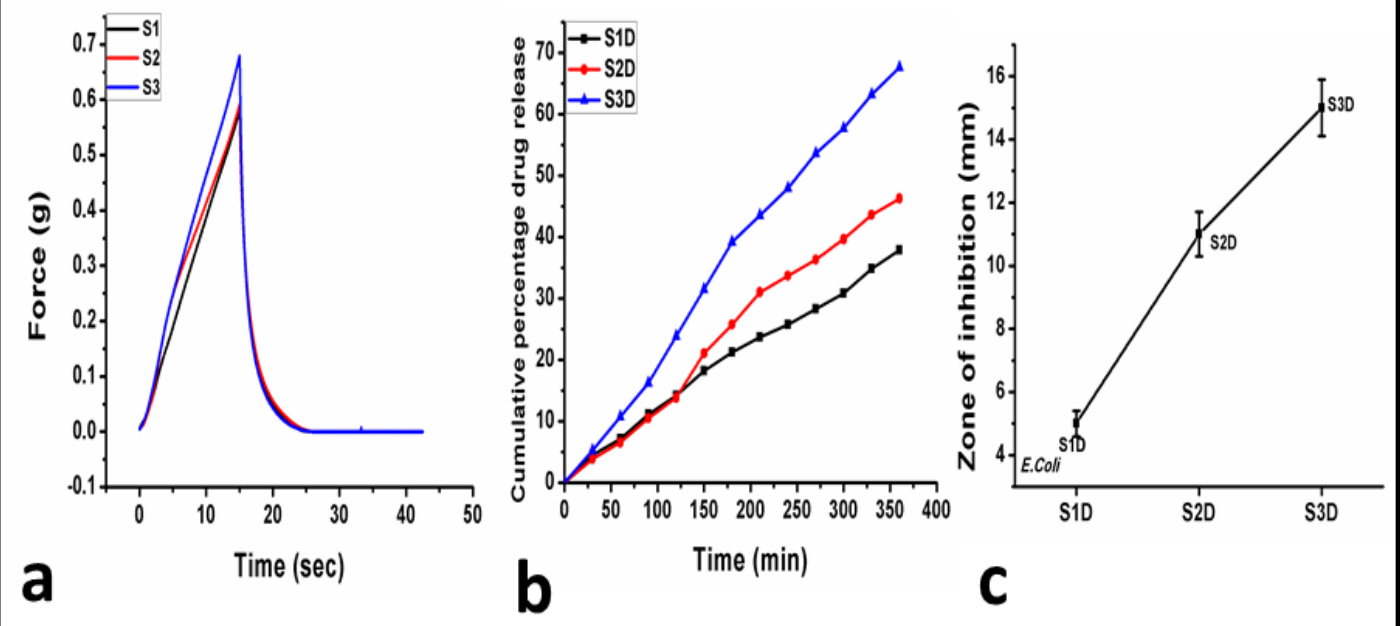

Figure 3. (a) Compressive profiles, (b) In Vitro drug release profiles, and (c) Zone of inhibitions (ZOIs) of hydrogels.

\section{Conclusions}

Hydrogel is a macromolecular polymer gel constructed of a network of cross-linked polymer chains. Chitosan Oligosaccharide is a biomaterial, water-soluble drug that has antibacterial properties and is used to prepare hydrogels for efficient drug delivery. Chitosan oligosaccharide is combined with carboxymethyl Cellulose to produce a hydrogel in different compositions for the best suitable option where the drug delivery and the biocompatibility are most efficient. 


\section{Funding}

This research received no external funding.

\section{Acknowledgments}

This study was supported by the Department of Biotechnology, KL University, Guntur.

\section{Conflicts of Interest}

The authors declare no conflict of interest.

\section{References}

1. Nie, J.; Pei, B.; Wang, Z.; Hu, Q. Construction of ordered structure in polysaccharide hydrogel: A review. Carbohydrate polymers 2019, 205, 225-235, https://doi.org/10.1016/j.carbpol.2018.10.033.

2. Sinha, A.; Kalambate, P.K.; Mugo, S.M.; Kamau, P.; Chen, J.; Jain, R. Polymer hydrogel interfaces in electrochemical sensing strategies: A review. TrAC Trends in Analytical Chemistry 2019, 118, 488-501, https://doi.org/10.1016/j.trac.2019.06.014.

3. Singh, B.N.; Veeresh, V.; Mallick, S.P.; Jain, Y.; Sinha, S.; Rastogi, A.; Srivastava, P. Design and evaluation of chitosan/chondroitin sulfate/nano-bioglass based composite scaffold for bone tissue engineering. International journal of biological macromolecules 2019, 133, 817-830, https://doi.org/10.1016/j.ijbiomac.2019.04.107.

4. Mallick, S.; Beyene, Z.; Suman, D.K.; Madhual, A.; Singh, B.N.; Srivastava, P. Strategies towards Orthopaedic Tissue Engineered Graft Generation: Current Scenario and Application. Biotechnology and Bioprocess Engineering 2019, 1-16, https://link.springer.com/article/10.1007/s12257-019-0086-6.

5. Sarkar, R.; Agrawal, A.; Ghosh, R. Preparation of ex-situ Mixed Sintered Biphasic Calcium Phosphate Ceramics from Its Co-Precipitated Precursors and Their Characterization. Transactions of the Indian Ceramic Society 2019, 78, 101-107, https://doi.org/10.1080/0371750X.2019.1619484.

6. Wang, X.; Lu, H.; Shi, X.; Yu, K.; Fu, Y.Q. A thermomechanical model of multi-shape memory effect for amorphous polymer with tunable segment compositions. Composites Part B: Engineering 2019, 160, 298305, https://doi.org/10.1016/j.compositesb.2018.10.048.

7. Neubauer, J.W.; Hauck, N.; Männel, M.J.; Seuss, M.; Fery, A.; Thiele, J. Mechanoresponsive hydrogel particles as a platform for three-dimensional force sensing. ACS applied materials \& interfaces 2019, 11, 26307-26313, https://pubs.acs.org/doi/10.1021/acsami.9b04312.

8. Huebsch, N. Translational mechanobiology: Designing synthetic hydrogel matrices for improved in vitro models and cell-based therapies. Acta biomaterialia 2019, 94, 97-111, https://doi.org/10.1016/j.actbio.2019.05.055.

9. Guder, D.G.; Krishna, M. Isolation and Characterization of Potential Cellulose Degrading Bacteria from Sheep Rumen. J Pure Appl Microbiol 2019, 13, 1831-1839, https://doi.org/10.22207/JPAM.13.3.60.

10. Suresh, L.; Brahman, P.K.; Reddy, K.R.; Bondili, J. Development of an electrochemical immunosensor based on gold nanoparticles incorporated chitosan biopolymer nanocomposite film for the detection of prostate cancer using PSA as biomarker. Enzyme and microbial technology 2018, 112, 43-51, https://doi.org/10.1016/j.enzmictec.2017.10.009.

11. Mahato, K.K.; Sabbarwal, S.; Misra, N.; Kumar, M. Fabrication of polyvinyl alcohol/chitosan oligosaccharide hydrogel: physicochemical characterizations and in vitro drug release study. International Journal of Polymer Analysis and Characterization 2020, 25, 353-361, https://doi. org/10.1080/1023666X.2020.1789382.

12. Kanikireddy, V.; Varaprasad, K.; Jayaramudu, T.; Karthikeyan, C.; Sadiku, R. Carboxymethyl cellulosebased materials for infection control and wound healing: A review. International Journal of Biological Macromolecules 2020, 164, 963-975, https://doi.org/10.1016/j.ijbiomac.2020.07.160.

13. Mallick, S.; Sagiri, S.S.; Behera, B.; Pal, K.; Ray, S.S. Gelatin-based emulsion hydrogels as a matrix for controlled delivery system. Materials and manufacturing processes 2012, 27, 1221-1228, https://doi.org/10.1080/10426914.2012.663133.

14. Sharma, P.; Jain, K.G.; Pandey, P.M.; Mohanty, S. In vitro degradation behaviour, cytocompatibility and hemocompatibility of topologically ordered porous iron scaffold prepared using 3D printing and pressureless microwave sintering. Materials Science and Engineering: C 2020, 106, 110247, https://doi.org/10.1016/j.msec.2019.110247.

15. Li, J.; Mooney, D.J. Designing hydrogels for controlled drug delivery. Nature Reviews Materials 2016, 1, 1-17, https://doi.org/10.1038/natrevmats.2016.71. 
16. Beyene, Z.; Ghosh, R. Effect of zinc oxide addition on antimicrobial and antibiofilm activity of hydroxyapatite: A potential nanocomposite for biomedical applications. Materials Today Communications 2019, 21, 100612, https://doi.org/10.1016/j.mtcomm.2019.100612.

17. Ammulu, M.A.; Tammina, K.; Bonigala, B.; Vemuri, D.P.K.; Podha, S.; Ronda, S.R. Fabricating multifunctional nanoparticles bonded to enzymatically oxidized fabrics for their various applications. Indian Journal of Fibre \& Textile Research (IJFTR) 2019, 44, 381-388, http://op.niscair.res.in/index.php/IJFTR/article/view/20858.

18. Belmar, L.; Toledo, L.; Sánchez, S.A.; Urbano, B.F. Fluorescent nanotubes in PHEMA hydrogels: Visualizing aggregation and distribution by confocal fluorescence microscopy. Materials Today Communications 2018, 16, 285-292, https://doi.org/10.1016/j.mtcomm.2018.07.002.

19. Amarnath, L.P.; Srinivas, A.; Ramamurthi, A. In vitro hemocompatibility testing of UV-modified $\begin{array}{lllll}\text { hyaluronan } \quad \text { hydrogels. } & \text { Biomaterials } & \mathbf{2 0 0 6}, & 27, & 1416-1424 \text {, }\end{array}$ https://doi.org/10.1016/j.biomaterials.2005.08.008.

20. Vemuri, P.K.; Dronavalli, L.; Nayakudugari, P.; Kunta, A.; Challagulla, R. Phytochemical Analysis and Biochemical Characterization f Terminalia Chebula Extracts For its Medicinal use. Biomedical and Pharmacology Journal 2019, 12, 1525-1529, https://doi.org/10.13005/bpj/1783.

21. Kante, R.K.; Somavarapu, S.; Vemula, S.; Kethineni, C.; Mallu, M.R.; Ronda, S.R. Production of recombinant human asparaginase from Escherichia coli under optimized fermentation conditions: effect of physicochemical properties on enzyme activity. Biotechnology and Bioprocess Engineering 2019, 24, 824832, https://link.springer.com/article/10.1007\%2Fs12257-019-0147-x.

22. Kante, R.K.; Vemula, S.; Somavarapu, S.; Mallu, M.R.; Gowd, B.B.; Ronda, S.R. Optimized upstream and downstream process conditions for the improved production of recombinant human asparaginase (rhASP) from Escherichia coli and its characterization. Biologicals 2018, 56, 45-53, https://doi.org/10.1016/j.biologicals.2018.10.002. 